\title{
Affective Correlates of Metropolitan Food Insecurity and Misery during COVID-19
}

\author{
Mayank Kejriwal, Ke Shen \\ University of Southern California \\ 4676 Admiralty Way, Suite 1001, Marina Del Rey, California 90292
}

\begin{abstract}
We explore the affective correlates of food insecurity and contrast with affective correlates of other variables, such as difficulty in paying household expenses, non-current payment status on rent and mortgage payments, loss (or expected loss) of employment income, and high likelihood of imminent eviction. Specifically, we compute associations between these variables recently analyzed and reported, in aggregate, by Bloomberg (and originally collected by the Census Bureau in February, 2021), and affective wellbeing data (collected by Gallup during 2020 after mass lockdowns in the United States). The data span fifteen metropolitan areas. In particular, we find (with 95 percent confidence) that loneliness is positively and highly correlated with food insecurity. Anger is highly correlated with several of the variables mentioned earlier, although not at a high enough significance. In this brief report, we report these preliminary associations and comment on possible implications, along with a set of research questions that are suggested by these early results.
\end{abstract}

Keywords: COVID-19, affective well-being, food insecurity, survey data, Census Bureau, Gallup

According to a report by the United States Department of Agriculture, food insecurity in America affected as many as 1 in 9 Americans (an estimated total

${ }^{\star}$ Both authors contributed equally. M. Kejriwal is the corresponding author.
Email address: $\{$ kejriwal, keshen $\}$ @isi.edu (Mayank Kejriwal, Ke Shen)

Preprint submitted to Elsevier

March 9, 2021 
of over 37 million Americans) even in pre-COVID days [1]. Post-COVID, the situation is much worse, with long lines at food banks and pantries considered 5 one of several defining images capturing the toll the crisis has taken on American families [2]. Food insecurity is just one of several 'misery' variables that have taken this toll; others, as we briefly describe in Materials and Methods, include job loss, threats of evictions, problems with paying rent or mortgage bills, and trouble with basic expenses.

In general, factors such as food insecurity, unemployment, and not being able to cover basic needs, are all known to cause significant declines in subjective well-being [3], 4]. In this preliminary study, we attempt to understand the affective correlates of a limited set of variables, most notably, food insecurity, in the context of fifteen metropolitan areas. Although the study is preliminary, we already find a strong and significant positive correlation between loneliness (an affect variable on which Gallup collects data at the individual level and that we aggregate at the metropolitan level) and food insecurity. We believe that this is an alarming consequence of the pandemic and has the potential to become a causal factor itself.

\section{Materials and Methods}

Gallup began fielding on March 13, 2020 by launching a specific survey that collected people's responses during the COVID-19 pandemic, polling daily random samples of the Gallup Panel (a probability-based, nationally representative panel of U.S. adults). As in other Gallup surveys (such as the Gallup World

25 Poll), questions measured aspects of subjective wellbeing (including affective wellbeing, which was the primary focus herein, and also life satisfaction), employment, health, and demographics, among others.

To measure affective wellbeing, the following was asked Did you experience the following feelings during a lot of the day yesterday with a Yes/No option for each of the following choices: Enjoyment, Worry, Sadness, Stress, Anger, Happiness, Boredom, Loneliness, Depression, Anxiety. This question is 
an individual-level affective wellbeing measurement, and the proportion of Yes responses for a feeling within a spatial unit (in this paper, a metropolitan area, summarized in Column 1 of Table 1 is taken as the measure of affective wellbeing for that spatial unit. We treat Happiness and Enjoyment as positive affect states and the others as negative affect states. The composite affective wellbeing score sums the scores for an individual on each measure, and ranges from -8 (with a Yes response on all eight negative affect choices and a No response on the two positive ones), to 2 . We aggregated all results at the level of the fifteen metropolitan areas being studied in this paper, discarding the rest.

The 'misery' variables that we consider in this paper are related to expenses and costs along various dimensions, loss of employment, and possibility of eviction. They were originally derived from Census Bureau data in February, 2021, but since we are interested in correlations at the metropolitan area, we consider 45 the rankings provided in a recent article by Bloomberg ${ }^{1}$. More details are provided both in that article, as well as the caption of Table 1 , which provides the rank of each city on all five misery variables.

One aspect of the study that must be noted herein is that the Gallup data aggregated over several months of 2020, and the more recent Census Bureau variables are slightly out of sync (by a few months). Therefore (and in conjunction with reasons grounded in statistics and empirical methodology), it is inappropriate to draw causal inferences. We also do not attempt a comparison with pre-COVID years to understand if the situation has gotten progressively worse over the last 1.5 years, as common experience and news would suggest. Formal exploration of these questions may be an important avenue for future research.

1 https://www.bloomberg.com/news/articles/2021-03-01/ houston-and-l-a-are-u-s-cities-where-pandemic-life-is-hardest 
Table 1: A ranking of 15 different metropolitan areas on each of five variables: (i) difficulty in meeting expenses (Expenses); (ii) expectation of imminent loss of employment of household member (Jobs); (iii) indication of whether there was either sometimes or often not enough to eat in the last 7 days (Food); (iv) indication of slight or no confidence that the household can pay next month's rent or mortgage on time (Housing); and, (v) indication that eviction or foreclosure in the next two months is either very likely or somewhat likely (Eviction). Lowerranked cities are worse off. Primary data is from the Census Bureau and the ranking was reported by Bloomberg.

\begin{tabular}{|l|c|c|c|c|c|}
\hline Metropolitan Area & Expenses & Jobs & Food & Housing & Eviction \\
\hline Seattle-Tacoma-Bellevue, WA & 14 & 14 & 14 & 14 & 10 \\
\hline Boston-Cambridge-Newton, MA-NH & 15 & 12 & 15 & 15 & 7 \\
\hline $\begin{array}{l}\text { Washington-Arlington-Alexandria, DC- } \\
\text { VA-MD-WV }\end{array}$ & 13 & 15 & 12 & 11 & 9 \\
\hline San Francisco-Oakland-Berkeley, CA & 12 & 7 & 13 & 12 & 13 \\
\hline Phoenix-Mesa-Chandler, AZ & 11 & 11 & 10 & 9 & 14 \\
\hline Dallas-Fort Worth-Arlington, TX & 10 & 9 & 5 & 10 & 6 \\
\hline $\begin{array}{l}\text { New York-Newark-Jersey City, NY-NJ- } \\
\text { PA }\end{array}$ & 6 & 6 & 8 & 7 & 12 \\
\hline $\begin{array}{l}\text { Philadelphia-Camden-Wilmington, PA- } \\
\text { NJ-DE-MD }\end{array}$ & 7 & 13 & 11 & 2 & 5 \\
\hline Riverside-San Bernardino-Ontario, CA & 3 & 2 & 3 & 13 & 15 \\
\hline Atlanta-Sandy Springs-Alpharetta, GA & 9 & 10 & 9 & 6 & 1 \\
\hline Chicago-Naperville-Elgin, IL-IN-WI & 8 & 8 & 6 & 5 & 3 \\
\hline $\begin{array}{l}\text { Miami-Fort Lauderdale-Pompano Beach, } \\
\text { FL }\end{array}$ & 2 & 4 & 1 & 8 & 11 \\
\hline Detroit-Warren-Dearborn, MI & 5 & 5 & 4 & 3 & 2 \\
\hline Los Angeles-Long Beach-Anaheim, CA & 4 & 1 & 6 & 4 & 4 \\
\hline Houston-The Woodlands-Sugar Land, TX & 1 & 3 & 2 & 1 & 8 \\
\hline
\end{tabular}


Table 2: Spearman's rank correlations between the five 'misery' variables in Table 1 *, **, and $* * *$ respectively indicate significance at the 90, 95 and 99 percent confidence levels.

\begin{tabular}{|l|l|l|l|l|l|}
\hline & Expenses & Jobs & Food & Housing & Eviction \\
\hline Expenses & $1.000^{* * *}$ & $0.832^{* * *}$ & $0.894 * * *$ & 0.657 & 0.046 \\
\hline Jobs & $0.832^{* * *}$ & $1.000^{* * *}$ & $0.777^{* * *}$ & 0.364 & -0.068 \\
\hline Food & $0.894 * * *$ & $0.777 * * *$ & $1.000 * * *$ & $0.502 *$ & 0.063 \\
\hline Housing & 0.657 & 0.364 & $0.502 *$ & $1.000 * * *$ & $0.550 * *$ \\
\hline Eviction & 0.046 & -0.068 & 0.063 & $0.550 * *$ & $1.000 * * *$ \\
\hline
\end{tabular}

\section{Results and Discussion}

As a first test of self-consistency, we provide the Spearman's rank correlation 2 between the variables in Table 2 . We find that, with the exception 60 of eviction, all variables are positively and strongly correlated to one another, with food, jobs and expenses being particularly self-consistent and significant. Housing and eviction seem to be exhibiting different behavior, which suggests that different cities may be experiencing different impacts due to city-specific issues and policies. The rankings in Table 1 seem to bear out this finding. The

65 Atlanta metropolitan area is the worst-performing (rank of 1) on eviction, and has a fairly low rank with respect to housing (6) but is otherwise doing (comparatively) well on expenses, jobs and food. The opposite (to an even greater extreme) is observed in the Riverside metropolitan area in California.

Table 3 tabulates our primary results. In general, we find that, between the positive (and composite) affective well-being measures, and a 'misery' variable, there is negative correlation, and the reverse is true for negative affective wellbeing measures. Diving deeper into the results, we find that the most striking

\footnotetext{
${ }^{2}$ This correlation is a better fit for this study both because Bloomberg ranked all 15 metropolitan areas rather than provide raw scales (Table 1), but also because of scaling issues that may cause problems with the (more traditionally used) Pearson's r correlation.
} 
result is the high positive correlation between the loneliness affect variable and food insecurity. Not only is the value high (0.521) but despite there being few data points $(\mathrm{N}=15)$, the correlation is significant at the 95 percent level or above, unlike the majority of the variables where only weak significance (or no significance) was obtained.

While causal factors cannot be teased out in this brief report (and the findings would need to be buttressed and replicated for greater certainty), these early results suggest that areas suffering the most from food insecurity are also more lonely. Considering the stigma that has historically existed (and often still exists) around the SNAP program [5] in the United States, perhaps this is not surprising, but the association between food insecurity and loneliness is alarming, especially given the unprecedented damage being caused by the COVID-19 85 crisis.

Beyond the strong and positive correlation between loneliness and food insecurity, the results suggest other intriguing research questions that could be addressed in future work:

1. Do the associations in Table 3 hold at an individual level?

2. Given increasing rural-urban divide in America, will similar findings hold for rural areas? If not, can we tease apart potential causal factors?

3. With increasing evidence that the COVID-19 pandemic has been particularly hard on both low-income and minority communities, do the associations presented earlier become even stronger and more significant for those communities? Furthermore, in what areas in the country do we observe particularly disparate impact?

\section{References}

[1] A. Coleman-Jensen, M. P. Rabbitt, C. Gregory, A. Singh, Household food security in the united states in 2018 (2019).

[2] T. Tully, Food lines a mile long in america's second-wealthiest 
Table 3: Spearman's rank correlations between misery variables and affective wellbeing variables. The composite well-being score is the difference between the sum of positive affect scores (Happiness, Enjoyment) and sum of negative affect scores (Stress, Anger, Boredom, Anxiety, Sadness, Worry, Depression). * ${ }^{* *}$, and *** respectively indicate significance at the 90, 95 and 99 percent confidence levels.

\begin{tabular}{|l|l|l|l|l|l|}
\hline & Expenses & Jobs & Food & Housing & Eviction \\
\hline Stress & 0.132 & 0.089 & 0.327 & -0.039 & -0.071 \\
\hline Anger & 0.227 & 0.043 & 0.394 & 0.420 & $0.453 *$ \\
\hline Boredom & -0.364 & -0.411 & -0.408 & -0.036 & 0.179 \\
\hline Anxiety & 0.071 & 0.195 & 0.247 & -0.147 & -0.050 \\
\hline Sadness & 0.261 & -0.018 & 0.395 & $0.504^{*}$ & $0.511 *$ \\
\hline Worry & 0.139 & -0.032 & 0.325 & 0.157 & 0.161 \\
\hline Depression & -0.124 & -0.165 & 0.002 & -0.141 & 0.252 \\
\hline Loneliness & 0.329 & 0.213 & $0.521 * *$ & 0.030 & 0.039 \\
\hline Happiness & 0.020 & 0.052 & -0.190 & 0.091 & -0.071 \\
\hline Enjoyment & 0.004 & -0.016 & -0.190 & 0.159 & -0.063 \\
\hline Composite & -0.136 & -0.039 & -0.352 & -0.064 & -0.214 \\
\hline
\end{tabular}



coronavirus-nj-hunger.html (2020).

[3] E. A. Frongillo, H. T. Nguyen, M. D. Smith, A. Coleman-Jensen, Food insecurity is associated with subjective well-being among individuals from 138 countries in the 2014 gallup world poll, The Journal of nutrition 147 (4) (2017) 680-687.

[4] R. Winkelmann, Unemployment, social capital, and subjective well-being, Journal of happiness studies 10 (4) (2009) 421-430.

[5] T. Gaines-Turner, J. C. Simmons, M. Chilton, Recommendations from snap participants to improve wages and end stigma, American journal of public health 109 (12) (2019) 1664-1667. 